\title{
An Ant Colony Algorithm for efficient ship routing
}

\author{
Ming-Cheng Tsou, Associate Prof. \\ Hung-Chih Cheng, Prof. \\ National Kaohsiung Marine University, Kaohsiung, Taiwan, R.O.C.
}

\begin{abstract}
With the substantial rising of international oil price and global warming on the rise, how to reduce operational fuel consumption and decrease air pollution has become one of the pursued goals of green ship. Ship route planning is an indispensible part of the ship navigation process, especially in transoceanic crossing ship routing. The soundness of ship routing not only affects the safety of ship navigation but also the operation economy and environmental protection. This research is based on the platform of Electronic Chart Display and Information System (ECDIS), and founded on Ant Colony Algorithm (ACA) combined with the concept of Genetic Algorithm (GA), to model living organisms optimization behaviour to perform efficient ship route planning in transoceanic crossing. Besides the realization of route planning automation, ship routing will achieve the goal of optimum carbon dioxide reduction and energy conservation, and provide reference for route planning decision.
\end{abstract}

Key words: ship routing; Ant Colony Algorithm; GIS; weather routing

\section{INTRODUCTION}

Almost $90 \%$ of the world's trade is carried by ship and, for the vast majority of this trade there is little or no alternative to transport by ship. Statistical data indicate that the freight carried by global commercial shipping has reached 8.919 million tons, and that this large volume has caused substantial negative environmental impacts, even though ships are the most environmentally friendly form of transportation. Still, it is estimated that 4-5 per cent of global $\mathrm{CO}_{2}$ emissions come from international shipping [6]. According to statistics, there are about 52,000 registered civil ships of more than 100t capacity, annually consuming 500 million tons of fuel, producing 1.6 billion tons of carbon dioxide and 30 million tons of sulfur dioxide, causing greater global warming and air pollution. Therefore, an additional option for reducing air pollution is to decrease fuel consumption during transit [3]. From a climatic and environmental perspective sustainability has become an issue of the highest priority, and this is an imperative that cannot and should not be ignored. Within the foreseeable future shipping will still be dependent on fossil fuels. We must develop energy efficient and environmentally friendly technologies encompassing shipboard energy production, ship propulsion and ship operation. In recent years the concepts of the "green ship" and "green shipping" have been raised in the international shipping industry. The green ship concept covers the full ship life cycle, and provides total solution to pollution prevention, noxious gas emission, and energy conservation problems associated with the ship's design, production, operation, and scrapping. Therefore, shipping management should take care not only of economic efficiency, but also environment protection to prevent pollution. The coordination of efficiency and environment allows for environmentally sustainable development. In order to achieve this goal, ships already in operation can be retrofitted with energy-conserving equipment but the most direct and economical method is to plan an optimum route. Choosing the optimum route, adjusting the speed and avoiding adverse weather can not only reduce fuel consumption significantly but also improve navigational safety.

The efficiency of transoceanic ship route planning is generally determined by the following three aspects [26]:

- the accuracy of the prediction of the ship's hydrodynamic behaviour under different weather conditions;

- the accuracy of the weather forecast;

- the capability and practicability of the optimization algorithm.

This paper deals with navigation technology and focuses on the third above mentioned aspect, i.e. ship route optimization. Because optimum route determination is a multi-criterion non-linear planning issue and encompasses many constraints, a balance between navigational safety and economic efficiency should be found [18]. In other words, the applied planning framework not only has to take care of navigational safetyrelated risk evaluation and deviation but also avoid total cost increase due to deviation measures. In order to achieve this optimization mission we made use of the new Ant Colony Algorithm (ACA) developed in the evolutionary computation field to model and simulate how ants choose foraging paths. 
We modified the ACA for marine navigation application, and integrated the Genetic Algorithm (GA) concept to improve its practical effectiveness. The geometric computation and information search function is provided by the Geographic Information System (GIS) - based platform ECDIS along with weather information for the background data required for ACA calculation. Through this research design we hoped to achieve optimum ship routing and use a more objective, quicker and more accurate model in order to:

automatically generate a more rational recommendation route that gives consideration to both safety and economy,

lighten the navigator's workload,

- provide a reference for route planning decision making,

- collaborate in the green shipping and environment sustainability effort.

\section{PREVIOUS WORK}

Since research in transoceanic crossing weather routing typically studies broader geographic domains, usually across the Atlantic or Pacific, it mainly considers mid- and short-term weather effects, especially the effects of wave direction and height on a ship's cross-ocean navigation speed, to achieve the goals of minimum sailing time and fuel consumption. After the appearance of an innovative paper by Hanssen and James [8], several methods have been proposed to solve the problem of minimizing sailing time. The major methods include:

1. Isochrone method. An Isochrone Line (Time-Front) is defined as the outer boundary of the attainable region from the departure point that is reached after a certain time. The isochrone method proposed by Hanssen and James [8] was used for a long time because it offered an easy manual calculation method. Hagiwara [7] devised a modified isochrone method suitable for computer programming. However this method can generate so-called "isochrones loops", and cannot ensure that the route will not cross land. Hence to counter these problems, Szlapczynska and Smierzchalski [19] made improvements to the isochrone method, consisting in generating the initial set of candidate routes necessary for evolutionary computation to enhance the effectiveness of initial random population generation in evolutionary computation. However this method utilizes bitmap-based algorithms and is not applicable to vectorbased ECDIS.

2. Calculus of variation. In Bijlsma publications $[1,2,3]$ application of the calculus of variation was extended to the minimization of fuel consumption. Kosmas et al. [11] used calculus of variation and a wave model to compute the optimum route and applied simulated annealing techniques, a type of optimization method, to accelerate algorithm convergence. This approach is limited to theoretical analysis, time-consuming in terms of calculation, and less applicable in practice [24]. Khalilov [10] states that the variation calculus method has no optimality proof.

3. Dynamic programming. Dynamic programming uses a grid system which divides a possible sailing region into several cells. Each crossing point of a cell boundary is a waypoint candidate. The dynamic programming solution algorithm seeks a ship's trajectory that is composed of the positions $(X, Y, T)_{k}, k=1,2, \ldots, N$, and the Control $C_{k}$ with the initial time $T_{0}$ and the initial ship position X [12]. Motte et al. $[14,15,16,17]$ conducted a series of detailed investigations on this issue, including grid mesh size setting, system development and design. In recent years, Wei et al. [25], Zhou and Chen [27] and Tang et al. [20] have also used dynamic programming to conduct automatic ship routing to reduce the workload of navigators. In Bijlsma paper [2] the relation between optimum control theory and dynamic programming was elucidated for the case of minimal fuel routing. The development of this method has become more mature but its weaknesses include the large quantity of nodes, large amount of space required for storage, inflexibility, and slow computational speed.

4. Network Model. A network is a special form of graph that consists of nodes and arcs. The network has numerical parameters such as length, cost and transit time. Lee et al. [12], through network modelling, used a depth-first search algorithm to combine weather modelling to establish a web-based optimum ship routing system. Montes [13] modelled the Optimum Track Ship Routing (OTSR) for U.S. Navy warships using a network graph of the Western Pacific Ocean. A binary heap version of Dijkstra's algorithm determines the optimum route for given model-generated wind and sea inputs. This method is similar to the dynamic programming method, but computation is inefficient because of a large quantity of nodes.

Problems such as difficult computerization, complicated and inefficient calculations, and easily getting trapped into local optima, are found in the above-mentioned traditional solution methods. With the development of artificial intelligence and the maturing of evolutionary computation technology, more and more researchers investigate model organism optimization by using evolutionary computation technology (for example: genetic algorithms (GA), ant colony algorithms (ACA) and particle swarm optimization) and apply it to route planning solutions. Tsou [22, 23], Smierzchalski and Michalewicz, [18] and Ito et al. [9] used the GA in the planning of collision avoidance and coastal navigation paths and gained very good results. The present research used the ACA as the research method, emulating ants' forage path finding behaviour, and applied it to search an optimum route. The two problems are similar with regard to their nature; ships are considered as ants, optimum navigation routes as forage paths, and weather conditions as obstacles. We hope that through this conversion approach the ACA calculation efficiency and global optimum search capability can be enhanced in achieving optimum ship routing.

\section{OPTIMUM SHIP ROUTING AND WEATHER ROUTING}

The ship weather routing develops an optimum track for ocean voyages based on ship's individual characteristics for a particular transit, and forecasts of weather and sea conditions. The advent of extended range forecasting and the development of selective climatology, along with powerful computer modelling techniques, have made ship routing systems possible [4].

\subsection{The principle of optimum ship routing}

Today, in the evaluation of optimum transoceanic routes, the primary concern, besides safety, is usually given to navigation time (or navigation speed) rather than to distance. In synthesized considerations of safety and energy conservation the shortest distance between two ports is not necessarily an optimum route. If there are many routes to choose from, under the condition that the main engine power outputs are equal, the route that takes the least amount of time is the optimum route. Shorter navigation time in itself implies less fuel consumption. 
Therefore, the optimum route must consider at least the following conditions:

- safety;

- avoiding severe weather and sea conditions as much as possible;

- natural factors such as weather, sea conditions and ocean currents, to increase navigational speed as much as possible.

In order to find the optimum route, when choosing and planning the navigation route and making corrections, the following principles must always be met:

- firstly, to ensure safety, avoid severe weather and sea conditions as much as possible;

- secondly, if, under the circumstances, there is no other option but to pass through a severe sea environment, pass in the least time possible;

thirdly, the design of navigation routes always aims to shorten navigation time and arrive ahead of the ETA (Estimated Time of Arrival);

- fourthly, as much as possible make use of natural conditions which help to increase navigation speed, such as down winds and down currents, and as much as possible avoid natural factors which work against speed of navigation, such as head seas.

The impact of wind and waves on ship speed is non-linear. In circumstances where other conditions are unchanged, their effect on ship's speed, main engine power outputs, and fuel consumption cannot be represented by a simple algebraic expression. Therefore, in terms of ship's energy conservation, if, upon planned corrections, navigation overall makes use of natural conditions that work to its advantage, and avoids natural conditions that work against it, total navigation time usually can be decreased, although the total navigation distance may be lengthened, because increased navigation speed can usually compensate for an increase in navigation distance.

\subsection{Computation of optimum route}

Based on the above-mentioned principles of optimum route planning, the cost of the optimum route is a function of the extent of obstruction and the distance between start and end points. Therefore, solving an optimum ship route problem may be seen as finding the route with the least cost among the set of candidate routes, while taking weather conditions into consideration. The mentioned cost is not limited to distance only but may include navigation time, fuel consumption or a combination of factors. The decision variables that should be controlled are the manoeuvring of course and speed. The total computational cost (I) is calculated as the sum of the costs over each route leg $(\mathrm{S})$. It is based on the ship's position $(\mathrm{P})$, changes in the manoeuvring control (C) (i.e., speed and course) and the time duration $(t)$. With these cost components, the ship routing problem can be described by the following formula: to minimize:

$$
I=\int_{S} f(P(s), C(s), t(s)) d s
$$

where:

$\mathrm{f}(\mathrm{P}, \mathrm{C}, \mathrm{t})$ - function of voyage cost for position, manoeuvring control and time; and $\mathrm{P} \in \mathrm{R}, \mathrm{C} \in \mathrm{C}_{\mathrm{A}}$

Let $\mathrm{R}$ be a possible sailing region. The manoeuvring control variable $C_{A}$ is the limit of the ship's output speed and the limit of course changing range. The cost per unit distance ds is measured by fuel consumption and degree of ship safety under sailing.

\section{ANT COLONY ALGORITHMS AND SHIP ROUTE COMPUTATION}

\subsection{The Ant Colony Algorithm principle}

The ant colony algorithm is a form of evolutionary computation that models the behaviour of real ants in search of food. The algorithm was proposed by Dorigo [5] and has been successfully used to solve many real-life problems, such as the travelling salesman problem (TSP). In the natural world, in ants' search of food, among many different possible routes from the nest to the food source, different ants will at first choose different paths but in the end nearly all ants will discover the same shortest path. This is because ants' search for the shortest path is an interactive process. They leave a certain amount of secretion (pheromones) on the path they pass, can sense the presence and intensity of the secretions and move toward the direction where the substance has a higher concentration. The amount of secretion increases with the increase in number of ants that pass by, and decreases in a certain function of time that passes by. The reason is that more ants pass by the shortest path and the speed of pheromone accumulation is higher than that of other paths. Through the continuous exchange of pheromones the optimum route between the nest and the food source is found in the end. The ACA is an optimization decision method designed according to this ability of ants in the natural world. One sets a certain number of artificial ants (ships) and follows a set of search rules to find the optimum route comprehensively. The objective of weather routing is to find the safest and most economical navigation route based on the weather and manoeuvring control conditions between the departure and destination points. The conditions and objectives are basically the same as those of the ACA.

\subsection{Division of the marine grid system}

In order to plan the necessary deviational and directional change in the navigation route, maritime space must first be divided into grids. The grids form the basis for waypoint candidates, and the navigation route structure consists of the waypoints (Fig. 1). Motte [16] has conducted detailed comparison and analysis regarding grid mesh size setting, and suggested that the grid mesh size should be four degrees longitude by a half degree latitude.

However the experimental measures are based on the North Atlantic Ocean from Western Europe to North America and may not be applicable to other marine areas. Additionally, given the case of a near north-south travel direction, the great circle route, using four degrees longitude as the grid's horizontal width, is not always appropriate. Therefore, in this study distance is used as the basis for the setting of grid size, hence flexibility for users themselves to change settings is saved. Essentially, navigation speed should be referred to, so that grid size corresponding to about the distance of one day's navigation, can be kept. The procedure to make a grid is as follows:

- first establish a great circle route between the starting point $\left(\mathrm{L}_{1}, \lambda_{1}\right)$ and end point $\left(\mathrm{L}_{2}, \lambda_{2}\right)$, to be the basis for route planning. The Great Circle route distance (D) and the initial course (C) can be obtained from the following formula:

$$
\begin{aligned}
& \operatorname{CosD}= \pm \operatorname{SinL}_{1} \cdot \operatorname{SinL}_{2}+\operatorname{CosL}_{1} \cdot \operatorname{CosL}_{2} \cdot \operatorname{CosDLo} \\
& \operatorname{CotC}=\frac{ \pm \operatorname{CosL}_{1} \cdot \operatorname{TanL}_{2}-\operatorname{SinL}_{1} \cdot \operatorname{CosDLo}}{\operatorname{SinDLo}}
\end{aligned}
$$

where:

DLo - the difference of longitude. 
When latitude $\mathrm{L}_{1}, \mathrm{~L}_{2}$ have same sign, then the signs of $\operatorname{Sin}_{1}$ $\mathrm{SinL}_{2}$ and $\operatorname{CosL}_{1} \mathrm{TanL}_{2}$ are (+); otherwise they are (-).

then set a distance $X$, and along the great circle route set segmentation points $(\mathrm{Lx}, \lambda \mathrm{x})$ at intervals of $\mathrm{X}$. The location $(\mathrm{Lx}, \lambda \mathrm{x})$ of a segmentation point from a departure point with a certain distance $\mathrm{D}_{\mathrm{x}}$ and initial course $\mathrm{C}$ is calculated by the formula:

$$
\begin{array}{r}
\mathrm{L}_{\mathrm{X}}=\operatorname{Sin}^{-1}\left(\operatorname{SinL}_{1} \cdot \operatorname{Cos}_{\mathrm{x}}+\operatorname{Cos}_{1} \cdot \operatorname{Sin}_{\mathrm{x}} \cdot \operatorname{Cos} \mathrm{C}\right) \\
\lambda_{\mathrm{X}}=\lambda_{1}+\operatorname{Cos}^{-1}\left(\frac{\operatorname{Cos}_{\mathrm{x}}-\operatorname{SinL}_{1} \cdot \operatorname{SinL}_{\mathrm{x}}}{\operatorname{Cos}_{1} \cdot \operatorname{Cos}_{\mathrm{x}}}\right)
\end{array}
$$

- then, using each segmentation point as a basis, extend the longitude line to the north and south and pick a set distance $Y$ to establish each segmentation point, so that the coordinates have the same longitude and different latitude;

- then connect these segmentation points in the north-south, east-west direction to form a grid system.

Finally set up the coordinate and node matrix, and the route planning will be established on this grid system. With advancements in communication and positioning technology the grid system and navigational route can be dynamically adjusted continuously, according to the latest weather conditions and the latest positioning point and end point on the great circle route.

\subsection{Implementation of Ant Colony Algorithm}

First, establish a grid system and its node matrix in the navigation area, and provide each node with the appropriate initial value in the matrix to form the initial node information concentration value matrix. Then, put all the ants on the starting point to allow them to move simultaneously toward direction of the aim, and to reach the destination point in the end (Fig. $1)$. In the process every ant uses the state transition rule and undergoes a selection process when gathering at the next node. Assuming that the time it takes for an ant to travel from node $a$ in the node set $i$ to any node $b$ in the next node set $i+1$, is the same, and distance is not a factor here, all the ants will reach the destination simultaneously and complete a circle. After all the ants have reached the destination, calculate the objective function value (Eq. 1) based on the routes discovered by each ant. Each node is also updated with overall information quantity, and nodes that are not passed by are treated as information quantity evaporation. Repeat this process until the optimum route is discovered. The detailed explanation of the process is as follows:

\subsubsection{Adjustment of information quantity}

The number of ants is assumed $\mathrm{m}$. And, every ant chooses the next route according to the corresponding probability based on the information quantity on the route, instead of choosing the route it has previously completed. Upon completing a route circle, it produces a corresponding quantity of information concentration, based on the total distance of the route, and updates the information concentration on the route just passed.

With $\tau_{\mathrm{ab}}(\mathrm{t})$ representing the information concentration on route $(\mathrm{a}, \mathrm{b})$ at $\mathrm{t}$-moment, the information concentration on this route at $\mathrm{t}+1$ moment is:

$$
\tau_{\mathrm{ab}}(\mathrm{t}+1)=\rho \cdot \tau_{\mathrm{ab}}(\mathrm{t})+\sum_{\mathrm{k}=1}^{\mathrm{m}} \Delta \tau_{\mathrm{ab}}^{\mathrm{k}}
$$

where:

$\rho \quad-$ a constant which indicates that the residual factor of information quantity is between 0 and 1 , and $(1-\rho)$ represents the information evaporation coefficient

$\Delta \tau_{\mathrm{ab}}^{\mathrm{k}}-$ the increased information quantity of ant $\mathrm{k}$ on route $(a, b)$ between $t$ and $t+1$ moment

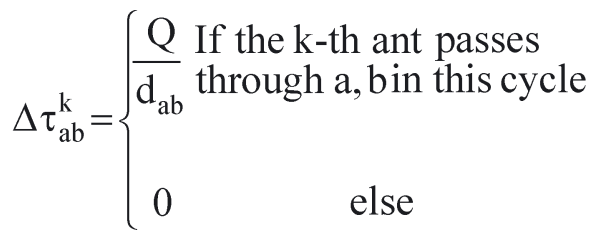

Eq. (7) indicates that the increased information concentration is related to the cost of the passed route, and:

$\mathrm{Q}$ is a constant which is the total information amount produced by each ant; $\mathrm{d}_{\mathrm{ab}}$ is the distance between node a and b, which can be calculated through Mercator Sailing, and then expressed in terms of other costs, such as time, fuel consumption etc.

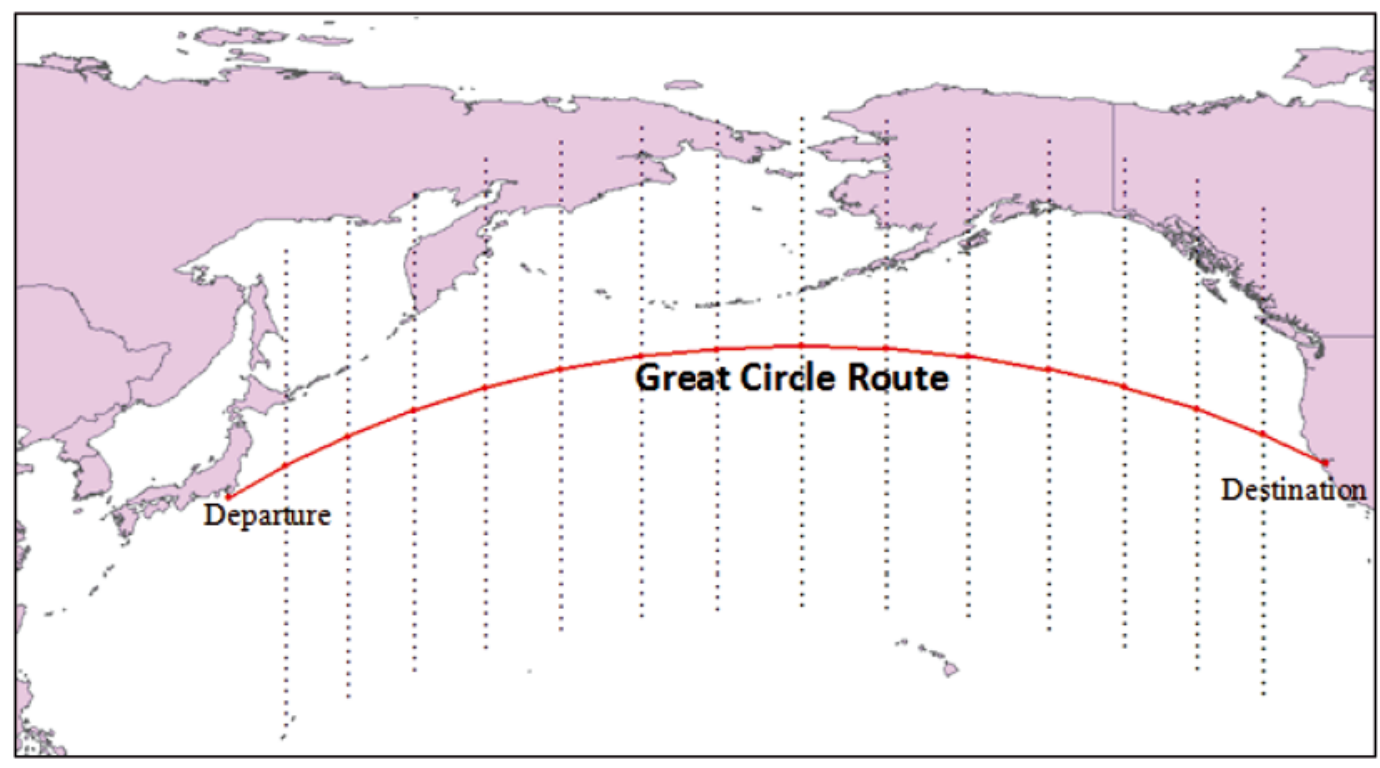

Fig. 1. Grid system and Great Circle Route 


\subsubsection{Waypoint selection principle}

The probability of the event that the ant $\mathrm{k}$ which is at node a will select node $\mathrm{b}$ as its next destination at $\mathrm{t}$ moment, is:

$$
\mathrm{P}_{\mathrm{ab}}^{\mathrm{k}}(\mathrm{t})=\left\{\begin{array}{cc}
\frac{\tau_{\mathrm{ab}}^{\alpha}(\mathrm{t}) \cdot \eta_{\mathrm{ab}}^{\beta}}{\sum_{\mathrm{b} \in \mathrm{N}_{\mathrm{k}(\mathrm{a})} \tau_{\mathrm{ab}}^{\alpha}(\mathrm{t}) \cdot \eta_{\mathrm{ab}}^{\beta}},} & \text { if } \mathrm{b} \in \mathrm{N}_{\mathrm{k}(\mathrm{a})} \\
0 & \text { else }
\end{array}\right.
$$

where:

$\mathrm{N}_{\mathrm{k}(\mathrm{a})}$ - the set of nodes that are yet to be visited by the $\mathrm{k}^{\text {th }}$ ant at node a

$\eta_{\mathrm{ab}}$ - heuristic information transferred from node a to node b. The information is obtained through the problem which has to be solved. In the problem the reciprocal of the cost of the points a and $b$, is $\eta_{a b}=1 / d_{a b}$

$\alpha \quad-$ the relevant importance degree of residual information element concentration

$\beta-$ the relevant importance degree of the expected value

\subsubsection{Calculation procedure}

The steps for using the ACA to determine the optimum route are listed below:

1. Initialize the information quantity on all the nodes on the navigation area, to form the initial information quantity matrix.

2. $m$ ants in number are ready to start at the starting point $A$.

3. Every ant selects the next node on the matrix based on the state transition rule of Eq. 8, and reaches the destination in the end, forming a feasible route.

4. Calculate the objective function of every ant's feasible route based on Eq. 1, and keep the optimum route.

5. According to the objective function and the information quantity adjustment principle of Eq. 6, adjust the information quantity of every point.

6. Check if iteration conditions are met (if the established iteration number or minimum objective function value is reached). If the conditions are met then the search is complete. If not, repeat the procedure starting from the step (2) until the iteration conditions are met.

\subsection{Calculation of navigation cost}

The calculation of navigation cost must take environmental conditions and ship performance conditions into account. To simplify the numerical experiment the amount of speed loss is calculated only by using the effect of waves.

\subsubsection{Ship response and performance}

Ships travelling in the ocean are affected by wind and waves. As a result the actual speed of the ship in waves will certainly be lower than that in calm water. The degree of speed loss will affect the calculation of the speed-weather conditions as well as computed navigation time or fuel consumption costs for the ship route [14]. Speed loss is the most important among the various factors involved in ship route calculations. It is present in many route calculation formulae and has significant influence on the precision of vessel positioning and the feasibility of route optimization by weather routing. In general, there are two approaches to studying ship speed loss. The first approach is to conduct seakeeping tank and wind tunnel model tests to simulate air and water resistance on the ship body and to derive theoretical expressions to quantify the effect of wave resistance on the ship. The second approach involves the use of mathematical statistics or neural network regression to obtain empirical formula for calculating ship speed loss due to waves, by means of a large database of weather observation information (e.g. weather records or logbook) and information regarding ship conditions at the time. The formulae for speed loss obtained from the two methods can be used to produce ship performance curves or speed loss curves, as shown in Fig. 2. The effect of wave direction on speed loss is different for head seas, following seas, and beam seas. The relationship between ship orientation and wave directions is demonstrated in Fig. 3. Based on ship performance curves in combination with sea condition forecast data such as wave height, wave direction, or wind speed, the impact of ship speed loss on the total navigational costs can be calculated. Empirical formulae for ship performance curves have been the subject of a number of studies in the past, where different factors were considered. The main factors affecting ship speed loss are wind and waves, and wave size is closely related to wind speed. For simplicity of formulation and because the present study is focused on route optimization algorithms, the empirical formula recommended by Soviet Central Maritime Research Institute to calculate ship speed loss has been selected as the basis for comparison. The included main factors are wave height, wave

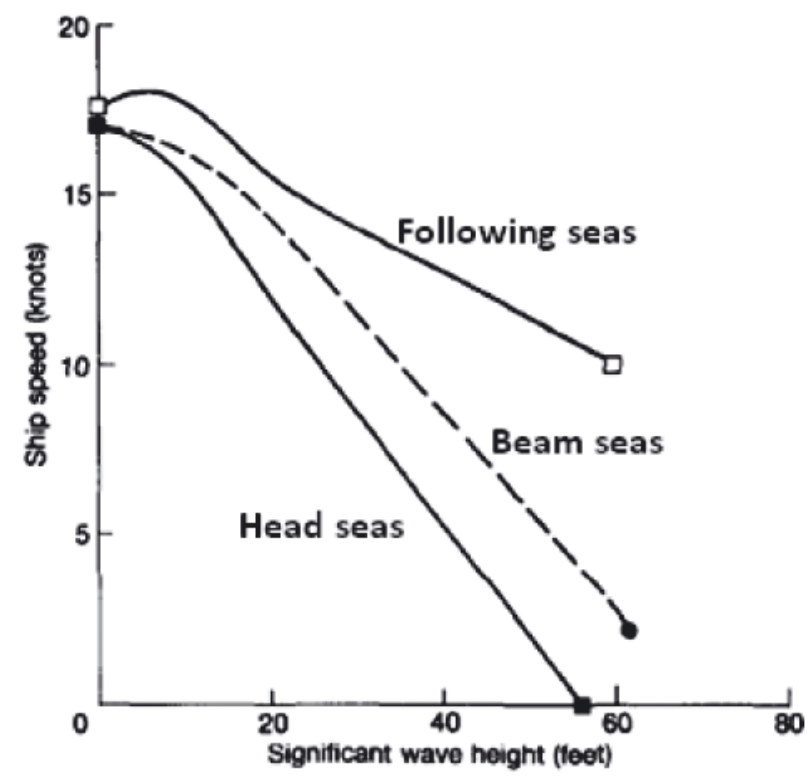

Fig. 2. James ship performance curves (Motte et al., 1988)

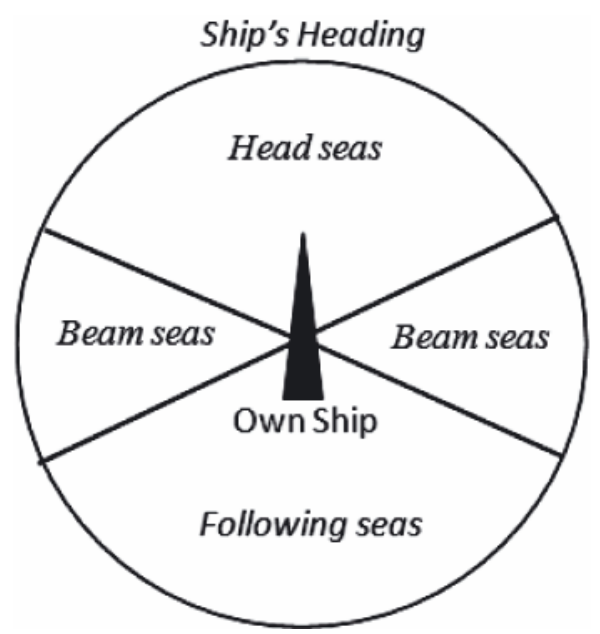

Fig. 3. Ship orientation and wave direction 
direction, and ship performance coefficient. If necessary, this can be substituted in the future by more sophisticated and accurate formulae. However, the assumed formula is sufficient for the purpose of validation of this research results. The formula is expressed as follows:

$$
\mathrm{V}=\mathrm{V}_{0}-(0.745 \mathrm{~h}-0.257 \mathrm{qh})\left(1.0-1.35 \times 10^{-6} \mathrm{DV}_{0}\right)
$$

where:

$\mathrm{V}$ - the actual speed in the sea

$\mathrm{V}_{0}-$ the speed in calm water

$\mathrm{h}$ - the wave height

q - the angle between ship heading and wave direction

D - the actual displacement of the ship (tons)

This formula is applicable to ships with displacement ranging from 5000 to 25000 tons, and speeds between 9 and 20 knots. Research has shown that when the wave height is between 0 and 5 meters, the difference between speed calculated by using this formula and the actual speed is less than 1 nautical mile. However, the calculated speed becomes slightly higher than the actual speed when the wave height is between 5 and 8 meters.

\subsubsection{Environmental data processing}

Environmental data include geographic, navigational, and weather data, i.e. the basic background information on navigation areas, and the primary basis for cost calculation. By means of intersection calculation and measurement of navigation distance and environmental information, different cost data can be obtained. The processing and calculation method is as follows: Geographic data. Land and ocean distribution and danger zone (e.g. shallow waters) distributions are stored as GIS's polygon vector data. With regard to land area, overlap analysis is conducted on the marine grid system's node and land distribution. Nodes in land areas are initialized with the value of 0 , while other nodes are set at a constant value. Thus, ants will not enter the nodes in the land area and will only search in the marine area.

- Weather data. With advances in information and communication technology in recent years, onboard internet connection by the INMARSAT ship station is often available and ample weather information is then easily accessible. Because ocean-going vessels can be only connected to the internet by satellites, which is relatively costly, only few ships use this method to gather weather information. However for the optimum route planning function in the Electronic Chart Display and Information System (ECDIS) the application of this method is an inevitable trend. In this study, the GRIB data downloaded from the internet is converted to a GIS format in the system and used for the route optimization.

The GRIB hydro-meteorological data is the standard defined by the World Meteorological Organization, and many service stations in the United States and Europe publish meteorological data in this format. In general, forecasts for the next 5 days are available, and they are updated every 6,12 or 24 hours. The spatial resolution can reach $0.5^{\circ} \times 0.5^{\circ}$. This format offers great flexibility in that wind speed, wind direction, air pressure, seawater temperature, wave height, wave direction, and some other meteorological information can be selectively included. Such information adequately meets the requirements of weather routing. The information can be downloaded directly from the internet to the computer onboard for further processing, saving time and improving accuracy. Because the loss of ship speed is mainly caused by waves and Eq. (9) is taken into consideration, in this study wave direction and wave height are extracted from the GRIB data for subsequent calculations. The wind effect was ignored because its relationship with waves is linear.

\subsubsection{Calculation method}

Wave direction and wave height grid data extracted from the GRIB are subsequently converted to cell layer data in the GIS to facilitate the integration with GIS computation. The weather cells are different from the nodes which constitute navigational waypoints in the navigation grid system. Next, the use of GIS's spatial analysis function is made to calculate the distance across all the cells that every candidate route passes, and find the wave height and wave direction data of the passed cells. By using ship speed loss formula the speed loss quantity can be computed. Finally, based on the distance across the cells, time/fuel consumption cost can be calculated. If navigation time is considered the primary cost, the computation formula is as follows:

$$
\begin{gathered}
\mathrm{t}_{\mathrm{j}}=\frac{\mathrm{d}_{\mathrm{j}}}{\mathrm{v}-\mathrm{w}_{\mathrm{j}}} \\
\mathrm{T}_{\mathrm{c}}=\sum_{\mathrm{j} \in \mathrm{J}}\left(\mathrm{t}_{\mathrm{j}}+\mathrm{d}_{\mathrm{j}} \times \mathrm{F}_{\mathrm{j}}\right)
\end{gathered}
$$

where:

$t_{j} \quad-\quad$ the required time for candidate route to pass the $j^{\text {th }}$ cell

$\mathrm{d}_{\mathrm{j}}-$ the distance for candidate route to pass the $\mathrm{j}^{\text {th }}$ cell

$\mathrm{V}$ - normal speed without weather disturbance

$\mathrm{W}_{\mathrm{j}}$ - the quantity of speed loss due to waves in the $\mathrm{j}^{\text {th }}$ cell

$\mathrm{T}_{\mathrm{c}}-$ total sailing cost

$F_{j}-$ additional unit distance cost factor in the $\mathrm{j}^{\text {th }}$ cell

\subsection{Improvements in computational performance}

\subsubsection{Limitations of maximum course deviation and maximum route segment length}

Because a great number of nodes in the vast ocean grid system is not feasible in relation to navigation planning without proper heuristic calculation, the process is very time-consuming and much calculation capacity will be wasted. Because of limitations of maximum course deviation and maximum route segment length much unnecessary route calculation results may be spared. The course deviation angle is limited to $\pm 60^{\circ}$ from the original course and the maximum route segment length cannot exceed that which can be covered in two days with the current speed. The selecting of appropriate values is a topic of further discussion.

\subsubsection{Determination of critical speed}

While performing ship routing calculations we cannot seek only the shortest navigation time and must also consider a ship's critical speed (allowed maximum speed) while navigating in storm conditions. Thus the calculation result can produce the optimum route on the premise of safety. The calculation of critical speed is mainly related to wave height and wave direction. In this study the following formula [24] is used to impose maximum speed limits on a ship's critical speed in waves.

$$
\mathrm{V}_{\text {Limit }}=\mathrm{e}^{0.13[\mu(\mathrm{q})-\mathrm{h}]^{1.6}}+\mathrm{r}(\mathrm{q})
$$

where:

$\mu(q)=12.0+1.4 \times 10^{-4} \mathrm{q}^{2.3}$

$\mathrm{r}(\mathrm{q})=7.0+4.0 \times 10^{-4} \mathrm{q}^{2.3}$ 
$\mathrm{q} \quad-\quad$ stands for the relative wave direction

$\mathrm{h}-$ stands for wave height

The formula reflects ship's critical speed under different wave conditions to ensure navigational safety.

\subsubsection{Limit on information quantity}

When the scale of the problem increases, the information quantity of the grid point which has never been searched, will decrease near to 0 , because of the information quantity evaporation coefficient (1- $\rho)$, decreasing the algorithm's global search capability. If $(1-\rho)$ becomes too small then the probability of a solution which has been searched, being selected, may become too large and will also affect the algorithm's global search capability [21]. Although increasing (1- $\rho)$ may enhance an algorithm's global search capability but also decrease the algorithm's rate of convergence. Thus, a range of information quantity $\left(\tau_{\min }, \tau_{\max }\right)$, is established, and if the information quantity exceeds this range the following corresponding limits are imposed:

$$
\tau_{a b}(t+1)=\left\{\begin{array}{lc}
\tau_{\min } & \tau_{a b}(t+1)<\tau_{\min } \\
\tau_{\max } & \tau_{a b}(t+1)>\tau_{\max } \\
\tau_{a b}(t+1) & \text { other }
\end{array}\right.
$$

\subsubsection{The addition of crossover operations to the genetic algorithm}

The crossover operation concept is added to the GA in this study. The crossover operation is processed through the searched optimum route and another ant's random route every time. If the two ants pass by the same grid point (waypoint), then a crossover operation is conducted between the two routes (excluding the starting and end points) based on the same grid point, namely, by combining the first half of route $\mathrm{A}$ with the second half of route $B$ to form a new route, and also the second half of route $\mathrm{A}$ and the first half of route $\mathrm{B}$ to form another new route. If the two ants do not pass the same grid point then crossover operations are not conducted. If, after the crossover operation, a route better than the current optimum route is obtained then the optimum route information is updated, otherwise, another crossover operation is performed. The adding of crossover operations increases the ant's ability to find a better route in a search, as well as variety of solutions.

\subsubsection{The addition of mutation operators to genetic algorithms}

In order to avoid local optima, in this study the concept of mutation operation was added to the GA through setting a certain probability value randomly selecting a grid point from the current optimum route and exchanging it with a notyet-passed random grid point from the optimum route, to form a new route. If the new route has a lower cost than that of the original optimum route then the original optimum route is replaced with this route.

\section{EXPERIMENT RESEARCH RESULTS}

\subsection{Condition settings of the experiment}

This experiment research simulated the navigation route from Yokohama to San Francisco, with $\left(34^{\circ} 40^{\prime} \mathrm{N}, 140^{\circ} \mathrm{E}\right)$ as the starting point coordinates, and $\left(37^{\circ} 45^{\prime} \mathrm{N}, 122^{\circ} \mathrm{W}\right)$ as the end point coordinates. The following conditions were applied to the simulation. The time spent in the algorithm is verified in the results.

- Weather conditions: the presumed weather conditions in the research had been previously ascertained. The weather cell size of wave height - wave direction weather data was set at latitude $0.5^{\circ}$ I longitude $0.5^{\circ}$. The GRIB data used was for the northern Pacific Ocean on 2011/11/25.

Conditions of course deviation and route segment length restriction: it was assumed that the ship was located at the $\mathrm{X}^{\text {th }}$ node (waypoint) in the node set $\mathrm{I}$, and the ship's next feasible node (waypoint) was confined within the range of $\mathrm{X} \pm 3$ node (waypoint) in the node set $\mathrm{I}+1$. This approach was used to provide the conditions of course deviation and route segment length restriction.

- Ship performance was simulated under the output power required to maintain the navigation speed of 18 knots in calm water. The speed loss curves, as in Eq. 9, were matched with wave height and wave direction data to simulate ship speed. In the simulation the ship displacement of 18000 tons was assumed.

- Grid system size: as shown in Fig. 1, the east-west direction interval distance along the great circle route was set at 300 nautical miles, with a total of 14 points. The distance along the longitude north-south direction line was set at 60 nautical miles $\left(1^{\circ}\right)$, with a total of 44 points. Thus, a grid system consisted of 616 waypoints was established. The route was generated through the ACA search; it was generated by testing and combining the waypoints.

- ACA parameter setting: $\alpha=3, \beta=2, \rho=0.5, Q=1000, \mathrm{~m}$ $($ number of ants $)=100, \mathrm{~N}$ (number of cycles) $=100$.

- Experiment environment: to perform the simulations PC of $4 \mathrm{~GB}$ memory and an Intel Core 2 Duo CPU $2.2 \mathrm{GHz}$ processor, was used. Visual Basic 2010 was used as the programming language and the geographic information feature provided by ArcGIS Engine's COM object was used to perform spatial analysis and geometry computation.

\subsection{Discussion of results}

The shipping routes were assessed on the basis of the total navigation time. From the experiments it was found that appropriate choice of the number of ants and the parameters can improve the efficiency of the algorithm. When the number of ants is small (ant no. 10), the ACA does not show clear signs of convergence, and an optimum solution is hard to obtain (as shown in Fig. 4). This is also the case when the ant number is larger (ant no. $=20$ ) and parameters $\alpha$ and $\beta$ are of low value (both $\leq 3$ ). When $\alpha$ and $\beta$ are larger (both $\geq 4$ ), convergence is more evident, but a large number of iterations is required. When ant no. $\geq 50$ and $\alpha, \beta$ both $>4$, a better convergence is achieved; however, the solution fell into a local optimum on many occasions (see Fig. 5).

It was found that when the number of ants is relatively large and the parameters are such that $\alpha<\beta(\alpha=2, \beta=3)$, better convergence was achieved through the ACA and a nearoptimum solution was more readily obtained. Therefore the parameters for deriving the optimum shipping route are set as follows:

$$
\alpha=2, \beta=3, \rho=0.5, \mathrm{~m}=200 \text { and } \mathrm{N}=100
$$

In addition, in order to validate the results the simulated results from east bound and west bound navigation were compared with those from navigation following the Great Circle Route. The performance of the simulation program under different weather conditions was observed. The simulated 


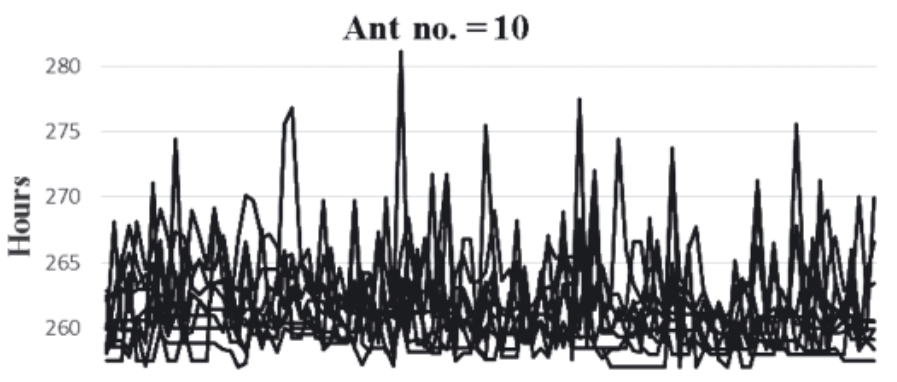

255

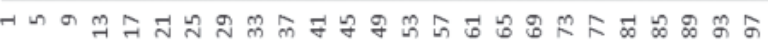

Iterations

Fig. 4. Poor convergence for a small number of ants. It is difficult to obtain a near-optimum solution. Notation: Ant no. stands for number of ants (Fig. 5)

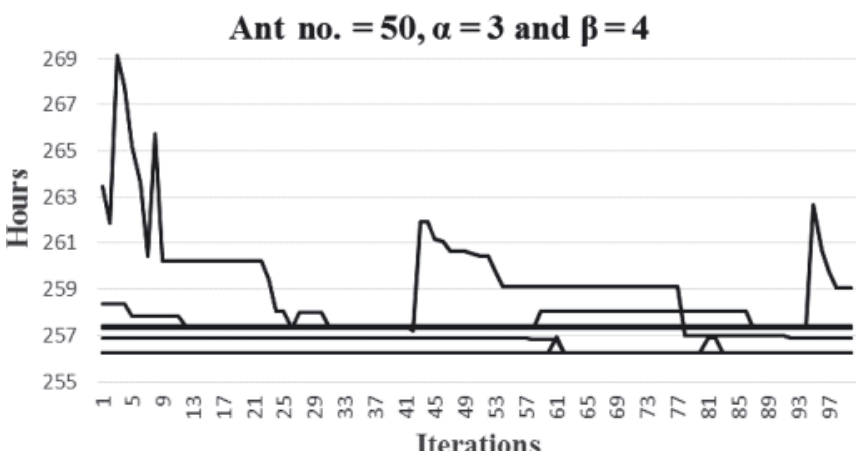

Fig. 5. When $\alpha=3$ and $\beta=4$, the solution achieved through $A C A$ is often the local optimum. Notation: Ant no. stands for number of ants

results are shown in Fig. 6 where the purple route is the Great Circle Route, the black route is the optimum route produced by the ACA, and the yellow routes are the alternative routes generated in the search process. During the east bound navigation, due to the influence of strong winds and stormy seas in the low pressure region, the ACA simulation suggests that the navigation route should be shifted south of the Great Circle Route (Fig. 7). If the ship followed the Great Circle Route for which the distance is 4518.6 nautical miles, and travelled with the calm water speed of 18 knots, the navigation time under the weather conditions was $261.8 \mathrm{~h}$ (Fig. 8) and the average speed was 17.26 knots. Although the distance generated by the ACA (equal to $4571.6 \mathrm{NM}$ ) is longer than that of the Great Circle Route, the navigation time was only $255.8 \mathrm{~h}$ and the average speed was 17.87 knots. Then $6 \mathrm{~h}$ and the corresponding amount of fuel can be saved compared with the Great Circle Route. For the west bound navigation, i.e. on the southern route, a ship encountered a head wind and head seas due to low pressure to the south of the Aleutian Islands. This casted an adverse effect on the navigation. The ACA algorithm therefore modified the route and caused it to deviate to the north off the Great Circle Route. The route entered the Bering Sea to the north of Aleutian Islands, and met favourable following seas and good weather, as shown in Fig. 9 and 10. In this case, the navigation time following the Great Circle Route was $267.2 \mathrm{~h}$ and the average speed was 16.91 knots. The route produced by the ACA had a longer distance of $4571.72 \mathrm{NM}$, but the navigation time was only $264.7 \mathrm{~h}$ (refer to Fig. 11) and the average speed was 17.27 knots. The saving of $2.5 \mathrm{~h}$ and the corresponding amount of fuel was achieved. The simulation results shows that the weather routing by means of the ACA makes use of favourable meteorological conditions and avoids to some extent encountering head seas and heavy wind regions, reducing this way the risk of ship damage and cargo loss. As regards the execution time, although algorithm efficiency for the entire program has not been optimized, the average execution time was about 5 min, which is sufficient for decision-making in real-time navigation.

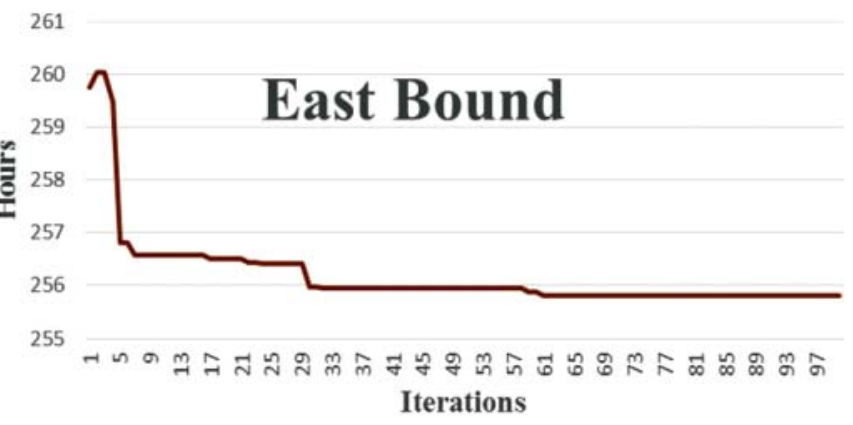

Fig. 8. Iteration history of optimum navigation time for east bound navigation

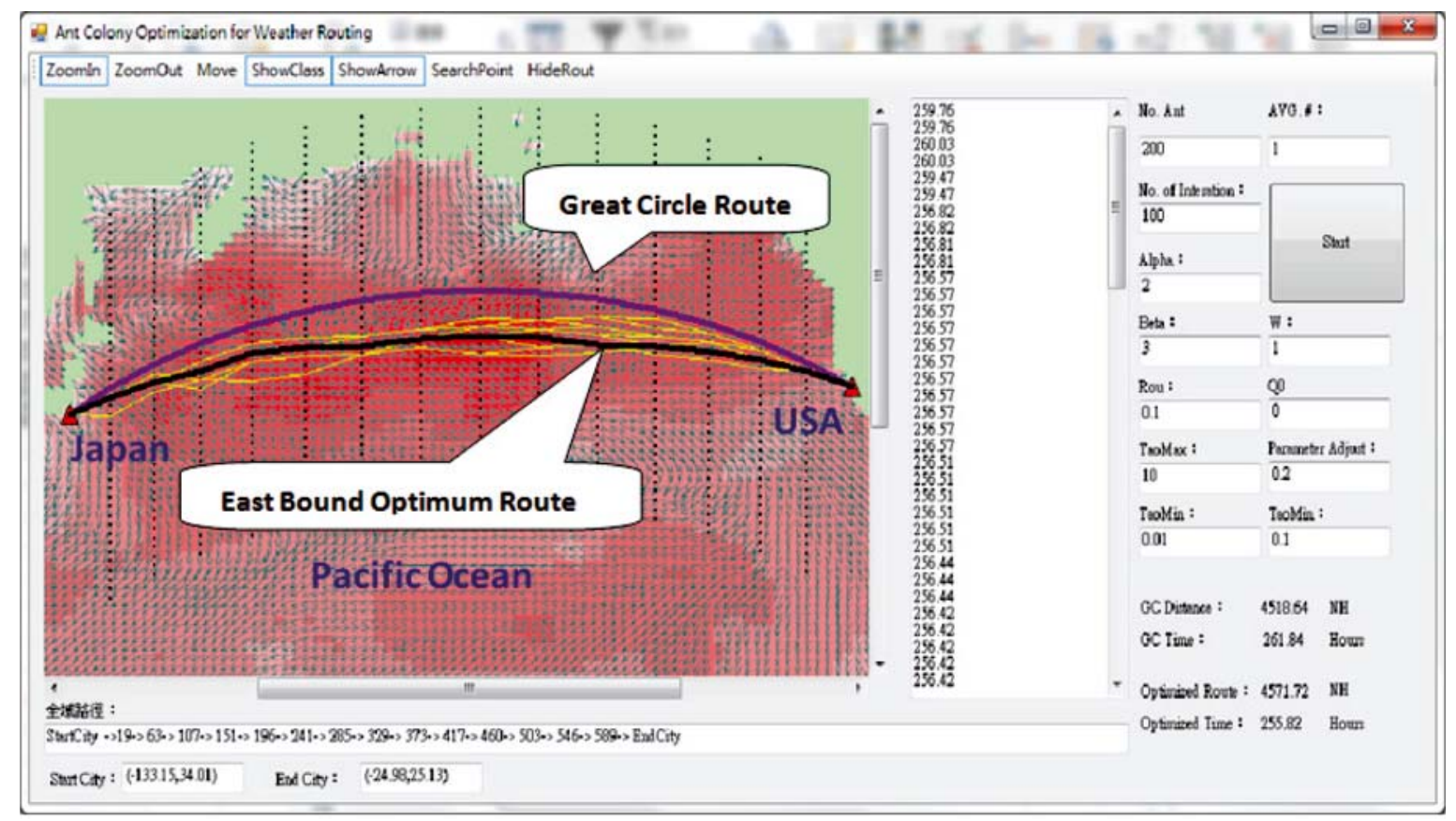

Fig. 6. Route simulation results for east bound navigation 


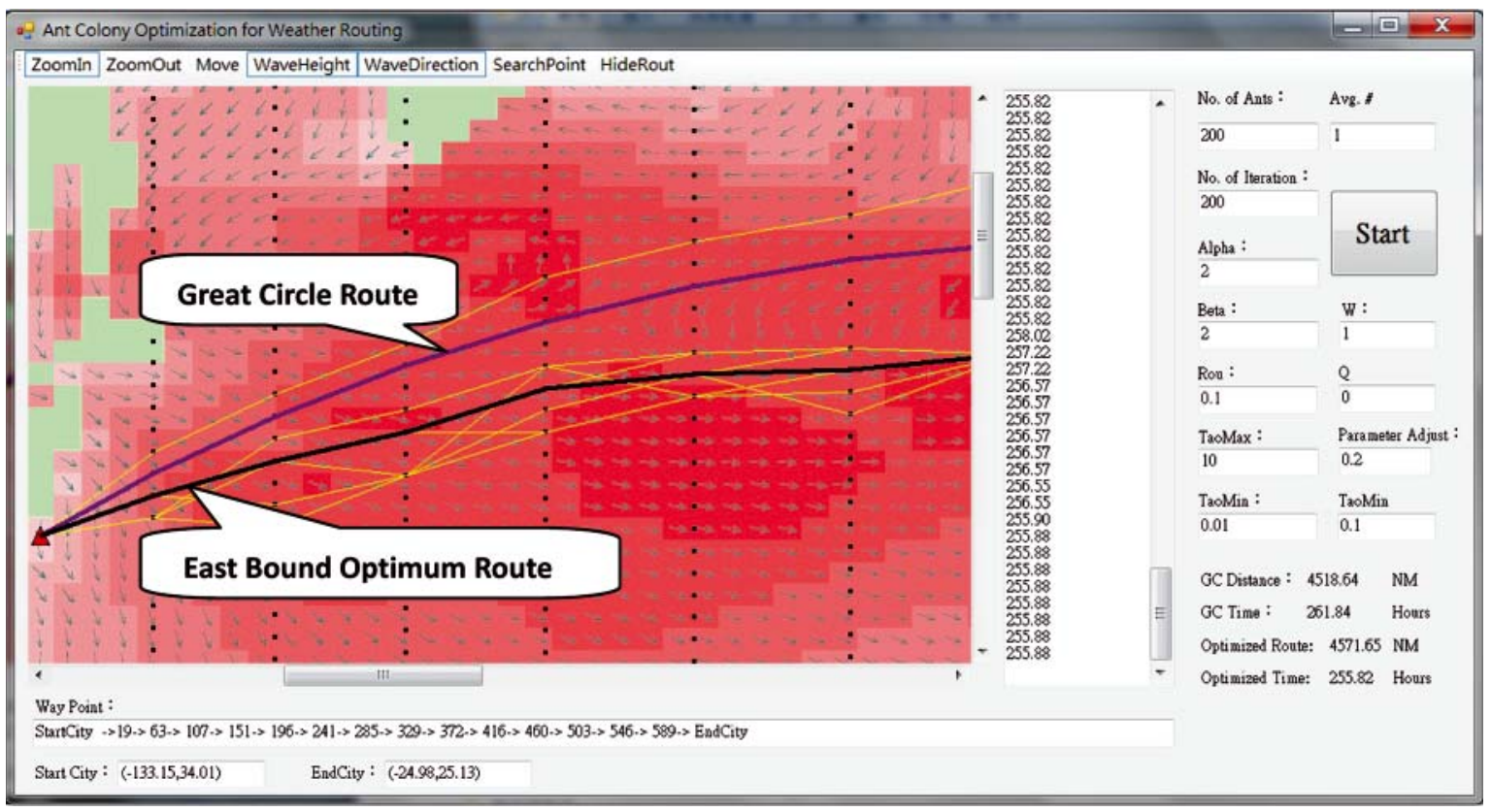

Fig. 7. Weather conditions and simulated route for east bound navigation

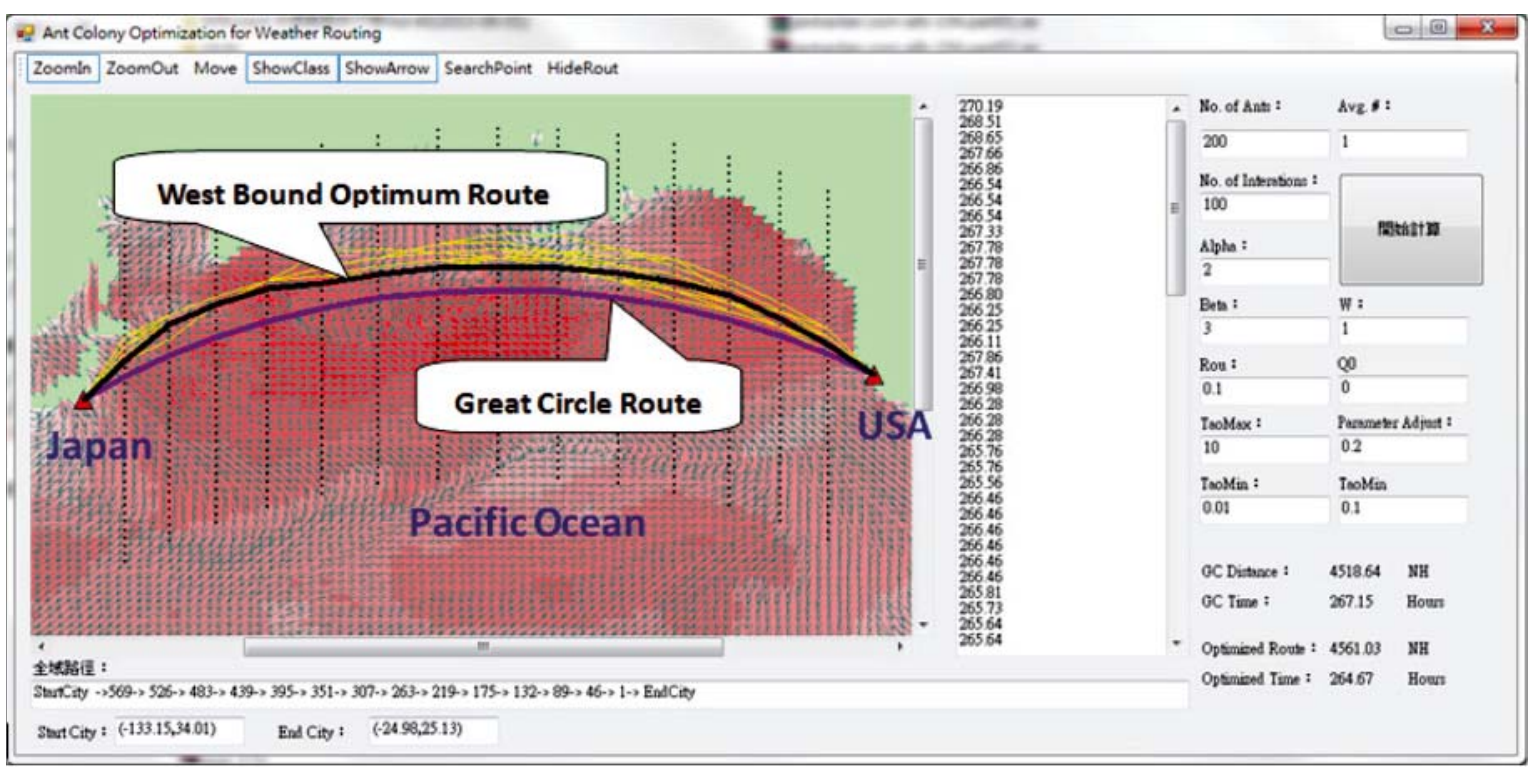

Fig. 9. Route simulation results for west bound navigation

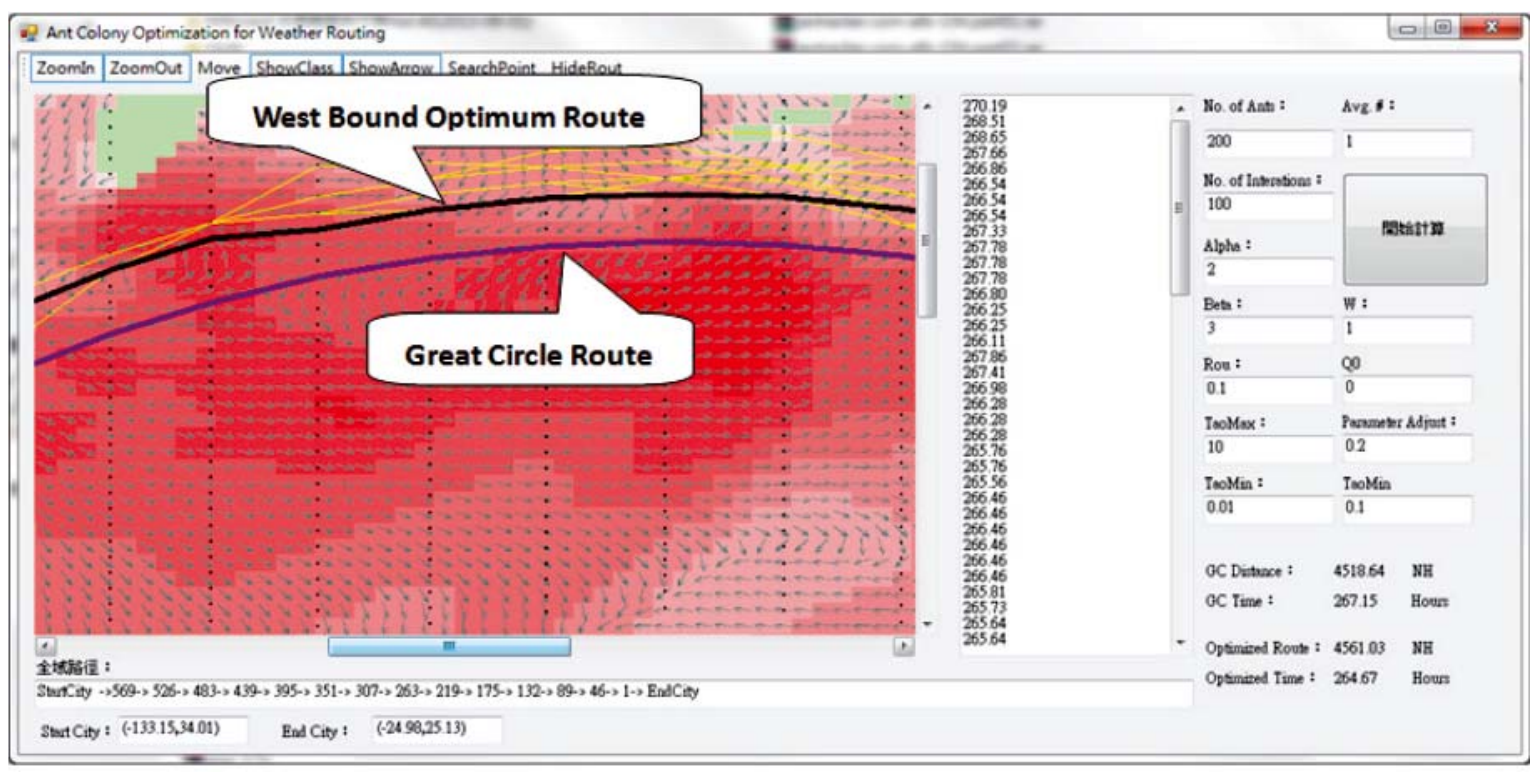

Fig. 10. Weather conditions and the simulated route for west bound navigation 


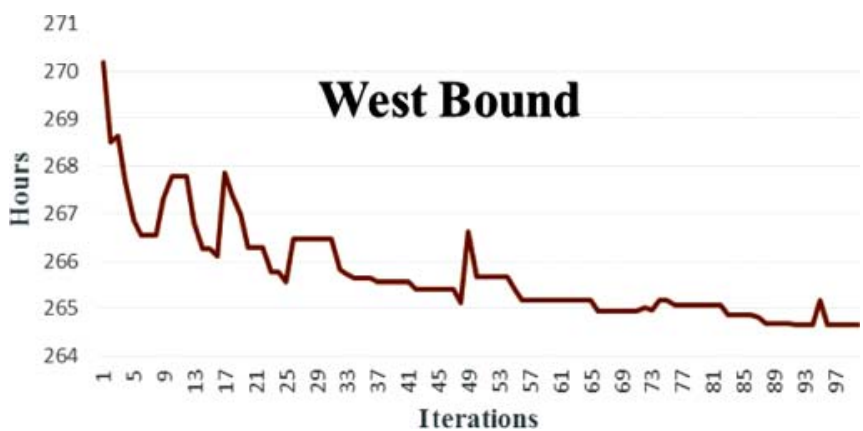

Fig. 11. Iteration history of optimum navigation time for west bound navigation

\section{CONCLUSIONS}

With the substantial rise in international oil prices, and global warming on the rise, to reduce operational fuel consumption and decrease air pollution has become one of the key goals of green shipping. In order to reach the goal, ships in operation can be fitted with energy-conserving equipment. However, optimum ship routing is the most direct and economical method. Therefore, application of decision support tools to help the routing of transoceanic crossings, not only can enable route planning automation, achieve the aim of carbon oxide reduction and energy conservation but also provide a reference for decision making in route planning. In this study a proper grid system and the improved ACA was used to establish a route searching model that simulates a living organism's optimum behaviour. In addition, GA's crossover and mutation computations, a ship's critical speed function, and GIS's spatial analysis and computation capability were integrated to automate the generation of a transoceanic crossing route, in consequence increasing route planning speed and quality, and lowering the ship crew's burden. With the growing popularity of distributed computing environments, many applications of parallel processing and distributed processing have been widely implemented. Considering the efficiencies brought about by distributed processes, the using of the distributed parallel processing structure together with the feedback mechanism of the ACA collective intelligence can further enhance ACA resolution efficiency. And, the computational speed will be higher than that of other resolution methods. Therefore, in the future the size of the grid system can be reduced to conduct more refined simulations by means of the combination of more waypoints. Also, in this study the candidate routes that pass through obstacles was removed without integrating an obstacle avoidance mechanism (such as island avoidance navigation). A relevant coastal navigation routing technique, suggested by Tsou [23], could be added in the future so that the results might better satisfy requirements of real time navigation decision support and enhance the energy conservation effect.

\section{Acknowledgements}

The authors would like to thank the National Science Council, Taiwan, R.O.C., for financial support under contract number: 100-2410-H-022-006.

\section{BIBLIOGRAPHY}

1. Bijlsma, S. J.,: A Computational Method for the Solution of Optimal Control Problems in Ship Routing. NAVIGATION, Journal of the Institute of Navigation, Vol. 48, pp. 145-154, 2001.

2. Bijlsma, S. J.: On the Application of Optimal Control Theory and Dynamic Programming in Ship Routing. NAVIGATION, Journal of the Institute of Navigation, Vol. 49, pp. 71-80, 2002.
3. Bijlsma, S. J.: Minimal Time Route Computation for Ships with Pre-Specified Voyage Fuel Consumption. The Journal of Navigation, Vol. 61, pp. 723-733, 2008.

4. Boditch, N.: The American Practical Navigator - 2002 Bicentennial Edition. National Imagery and Mapping Agency, U.S. Government, 2002

5. Dorigo, M., Manizzzo, V. and Colomi, A.: Ant system optimization by a colony of cooperating agents. IEEE Transaction on System, Man and Cybernetics- Part B: Cybernetics, Vol. 26, No. 1, pp. 29-41, 1991.

6. Flecks, J.: Study Green Shipping. HVB Global Shipping, Hamburg, pp. 4-5, 2009.

7. Hagiwara, H.:Weather Routing of Sail Assisted Motor Vessels. Ph. D. Thesis, Delft University, Holland, 1989.

8. Hanssen, G. L. and James, R. W., "Optimum Ship Routing," The Journal of Navigation, Vol. 13, pp. 253-272, 1960.

9. Ito, M., Zhang, F. and Yoshida, N.: Collision avoidance of ship with genetic algorithm. Proceedings of 1999 IEEE International Conference on Control Applications, pp. 1791-1796, 1999.

10.Khalilov, S. I.: Stochastic dynamic programming method for computing the most advantageous ship navigation routes. Meteoro. Hydrol., No. II, 1980.

11.Kosmas, O. T., Vlachos, D. S. and Simos, T. E.: Obstacle Bypassing in Optimal Ship Routing Using Simulated Annealing. Proceedings of International Electronic Conference on Computer Science, Vol. 1060, pp. 79-82, 2008.

12.Lee, H., Kong, G. \& Kim, S.: Optimum Ship Routing and It's Implementation on the Web. Lecture Notes in Computer Science, Vol. 2402/2002, pp. 11-34, 2002.

13.Montes, A. A.: Network Shortest Path Application for Optimum Track Ship Routing, Master Thesis, U.S. Naval Postgraduate School, Monterey, California, 2005.

14.Motte, R. Burns, R. S. and Calvert, S.: An Overview of Current Methods Used in Weather Routeing. The Journal of Navigation, Vol. 41, No. 1, pp. 101-114, 1988.

15.Motte, R. and Calvert, S.: Operational Considerations and Constraints in Ship-based Weather Routeing Procedures. The Journal of Navigation, Vol. 41, No. 3, pp. 417-433, 1988.

16.Motte, R. and Calvert, S.,: On The Selection of Discrete Grid Systems for On-Board Micro-based Weather Routeing. The Journal of Navigation, Vol. 43, No. 1, pp. 104-117, 1990.

17.Motte, R., Fazal, R., Epshteyn, M. Calvert, S. and Wojdylak, H.: Design and Operation of a Computerized, On-Board, Weather Routeing System. The Journal of Navigation, Vol. 47, No. 1, pp. 54-69, 1994.

18.Smierzchalski, R., Michalewicz, Z.: Modeling of ship trajectory in collision situations by an evolutionary algorithm. IEEE Transactions On Evolutionary Computation, Vol. 4, pp. 227-241, 2000.

19.Szlapczynska, J., Smierzchalski, R.: Adopted Isochrone Method Improving Ship Safety in Weather Routing with Evolutionary Approach. International Journal of Reliability Quality and Safety Engineering, Vol. 14, No. 6, pp. 635-646, 2007.

20.Tang, X.-T., Fen, G.-S., Zhao, W.-F.: Application of Dynamic Programming in Designing Ship's Optimum Route, in Chinese. Journal of Guangzhou Maritime College, Vol. 17, No. 2, pp. 1820, 2009.

21.Tian, W. and Zhan, A.,: Research on Path Planning for UCAV based on Improved Ant Colony Algorithm, in Chinese. Fire Control and Command Control, Vol. 33, No. 11, pp. 69-72, 2008.

22.Tsou, M.-C., Kao, S.-L., Su, C.-M.: Decision Support for Genetic Algorithms for Ship Collision Avoidance Route Planning. The Journal of Navigation, Vol. 63, pp. 167-182, 2010.

23.Tsou, M.-C.,: Integration of a Geographic Information System and Evolutionary Computation for Automatic Routing in Coastal Navigation. The Journal of Navigation, Vol. 63, pp. 323-341, 2010.

24.Wang, F., Jia, C.: The Study on The Optimal Ship Routing, in Chinese. Journal of Dalian Maritime University, Vol. 24, No. 2, pp. 61-64, 1998. 
25.Wei, X., Yu, Z., Wang, Z.: Design of Optimum Ship Route Based on Dynamic Programming, in Chinese Navigation of China, Vol. 57, pp. 16-18, 2003.

26. Wei, S., Zhou, P.: Development of a 3D Dynamic Programming Method for Weather Routing. International Journal on Marine Navigation and Safety of Sea Transportation, Vol. 6, No. 1, pp. 79-83, 2012.

27.Zhou, P., Chen, H.-W.: Improved Calculation Method of the Shortest Time Route, in Chinese. Science Technology and Engineering, Vol. 18, No. 21, pp. 5876-5880, 2008.

\section{CONTACT WITH THE AUTHORS}

Ming-Cheng Tsou*), Associate Prof. Department of Shipping Technology, National Kaohsiung Marine University, Kaohsiung, Taiwan, R.O.C.

Hung-Chih Cheng, Prof.

Department of Marine Environment and Engineering, National Sun Yat-sen University, Kaohsiung, Taiwan, R.O.C.

*)Author for correspondence: Ming-Cheng Tsou (e-mail: d86228006@yahoo.com.tw) 